\title{
LABORATORIO REMOTO VISIR COMO HERRAMIENTA PEDAGÓGICA
}

VÁZQUEZ, Raimundo; BURGOS, Alejandro (*)

\section{RESUMEN}

Se describe una experiencia en el área de Electrotecnia. El objetivo principal fue facilitar el proceso de aprendizaje del alumno y mejorar la práctica docente mediante la utilización de estrategias basadas en la indagación y la experimentación. Para tales fines se articularon aspectos formales y teóricos de electricidad y fenómenos físicos. Se partió del supuesto que al sujeto de aprendizaje que se inicia en la Ingeniería le resulta complejo relacionar leyes fundamentales como ley de Ohm o Kirchhoff. A fin de poder facilitar el aprendizaje de conceptos relacionados con electrotecnia, se utilizó un laboratorio remotoque permitió analizar comportamientos de señales eléctricas y contrastar con los resultados teóricos y prácticos.

Esta modalidad de aprendizaje impacta tanto en el rol del docente como también en el del alumno, el docente se convierte en un guía o asesor encargado de sugerir posibles caminos y soluciones a problemas planteados, por otro lado, debe diseñar material didáctico orientado a la experimentación mediante el uso de instrumentos de medidas eléctricas para desarrollar habilidades relacionadas con el uso de ambientes de laboratorio.

Palabras clave: estrategias didácticas, laboratorio remoto, herramienta pedagógica.

\section{INTRODUCCIÓN}

La idea central de este trabajo es contribuir con el proceso de enseñanza aprendizaje de los fundamentos de electrotecnia en las carreras de Ingeniería Electromecánica, Ingeniería Química e Ingeniería en Sistemas de Información. Se busca generar recursos didácticos que permitan al docente mejorar su práctica y facilitar en el aprendiz la apropiación de conceptos de electrotecnia utilizando la herramienta pedagógica denominado laboratorio remoto.

\section{CONCEPTOS FUNDAMEN- TALES \\ A. Laboratorio Remoto Como Recurso Pedagógico \\ Los laboratorios reproducen la natu-}

(*) Universidad Tecnológica Nacional Facultad Regional Resistencia. Correo: ray_vazquez_2005@hotmail.com 
raleza y principios por los cuales las cosas funcionan y ofrecen al alumno para su observación, comprensión y modificación. Los laboratorios son la base en la formación del ingeniero, y su uso intensivo asegura buenos profesionales y sus competencias [1][2][3]. El laboratorio permite desarrollar habilidades que el alumno debe adquirir en el grado; y buena parte de ellas solo pueden ser obtenidas en dicho ambiente. Es especialmente importante no relacionar exclusivamente el trabajo del alumno en el laboratorio con las prácticas durante el horario de cursado. Los laboratorios deben estar siempre a disposición del alumno de ingeniería para desarrollar y potenciar sus habilidades. En este sentido el laboratorio remoto promueve el aprendizaje autónomo y significativo del alumno. Dicha tarea nunca fue fácil, debido a distintos aspectos relacionados a la creación, mantenimiento de los laboratorios y cómo integrarlo en un ambiente académico. Los problemas son organizativos (espacios, horarios, monitores, profesores, etc.), económicos (equipos, mantenimiento, averías, etc.), didácticos (gestión de prácticas y proyectos, actitud de los alumnos, alumnado disperso, etc.). Los laboratorios remotos vienen a relajar algunos de los problemas anteriores, pero en ningún caso sustituyen a los laboratorios clásicos. Un laboratorio remoto permite que el sujeto del aprendizaje y el profesor trabajen desde su casa como si estuvieran en el laboratorio de la universidad. En ese sentido un laboratorio remoto es muy útil y se convertirá en una herramienta de enseñanza aprendizaje y mejorará la calidad de enseñanza del profesor.

\section{B. VISIR como herramienta pedagógica}

VISIR (Virtual Instruments Systems In Reality) es un laboratorio remoto que ha sido desarrollado en el Instituto de Tecnología de Blekinge de Suecia, de enorme difusión a nivel mundial [4][5][6]. El proyecto se encuentra en desarrollo y es llevado a cabo por un consorcio de instituciones de educación superior, europeas y latinoamericanas, con posibilidad de incorporar adherentes. Destacan los objetivos estratégicos y de innovación, tanto en lo tecnológico como en lo educativo, alcanzables mediante la colaboración, a los fines de generar, por un lado, cambios metodológicos en la enseñanza de temas centrales en carreras de Ingeniería y por otro la ampliación de posibilidades de experimentación remota compartiendo recursos.

\section{Laboratorio Remoto VISIR}

Existen muchas herramientas para simular un circuito electrónico, en ese sentido la idea del VISIR es implementar un circuito real remotamente, por lo cual se considera una gran iniciativa para la educación a distancia teniendo en cuenta lo que lleva este tipo de educación de ventajas como ahorro de coste, tiempo y menos riesgo, ya que el alumno no entra en contacto físico con los instrumentos reales, sin embargo, está trabajando con ellos. La iniciativa de los laboratorios remotos nació en el instituto de tecnología de Massachusetts con su proyecto iLAB que pretende trabajar con circuitos electrónicos remotamente [7]. Como se ha mostrado, el VISIR es capaz de sustituir las prácticas presenciales totalmente, por 
lo cual era una gran iniciativa del departamento de Ingeniería Eléctrica, Electrónica y Control Industrial de la UNED el aplicarlo en las asignaturas de Circuitos Eléctricos y Electrónicos. Se trata específicamente de la adquisición de una innovación educativa que permite facilitar el proceso de aprendizaje en áreas de la tecnología como por ejemplo electrónica y electricidad elemental.

\section{Procedimiento}

Para realizar las prácticas se deben tener en cuenta cuatro momentos fundamentales.

El primer empieza cuando el profesor y los alumnos realizan el proceso de registro en la página WEB del VISIR.

Existen tres tipos de cuentas: Cuenta de profesor, Cuenta de alumnoy Cuenta de invitado.

En la figura 1 se muestra a un grupo de doce alumnos de la carrera de Ingeniería Electromecánica de tercer año participantes de la experiencia del laboratorio remoto.

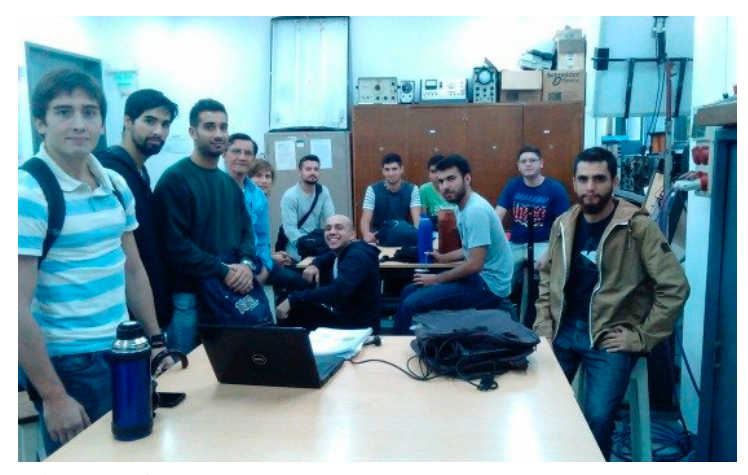

Fig. 1: Alumnos participantes de la experiencia del laboratorio remoto.

El segundo momento comienza cuando el docente responsable del laboratorio desarrolla los fundamentosteóricos prácticos por los cuales es posible realizar en forma remota conexiones de dispositivos electrónicos en un protoboar virtual y obtener medidas eléctricas reales, como se observa en la figura 2.

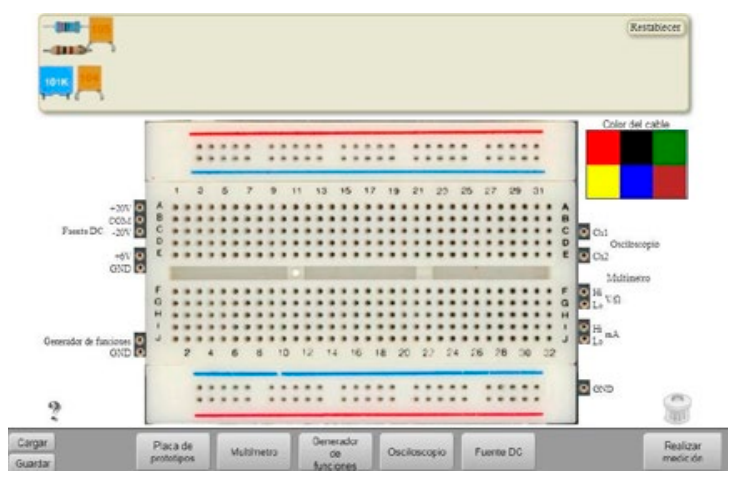

Fig. 2: Protoboar virtual del laboratorio remoto.

Esta etapa es muy importante porque muestra la diferencia más significativa entre los laboratorios virtuales y el laboratorio remoto VISIR.

E1 tercer momento se concentra en el sujeto del aprendizaje. El mismo debe incorporar conocimiento mediante tutoriales en la WEB del VISIR y videos informativos.

El alumno desarrollaen esta etapa competencias relacionadas al uso de instrumentos, toma de medidas eléctricas y análisis de señales.

Los instrumentos accesibles son: multímetro digital, un osciloscopio, generador de funciones y una fuente de tensión eléctrica como se muestra en la figura 3.

Una vez adquirida la destreza necesaria en el manejo de los instrumentos por parte del alumno, el docente pasa a desarrollar la última etapa.

En este sentido el VISIR en su página WEB tiene unas series de guías de laboratorios conceptuales relacionados a electrónica o electrotecnia. 

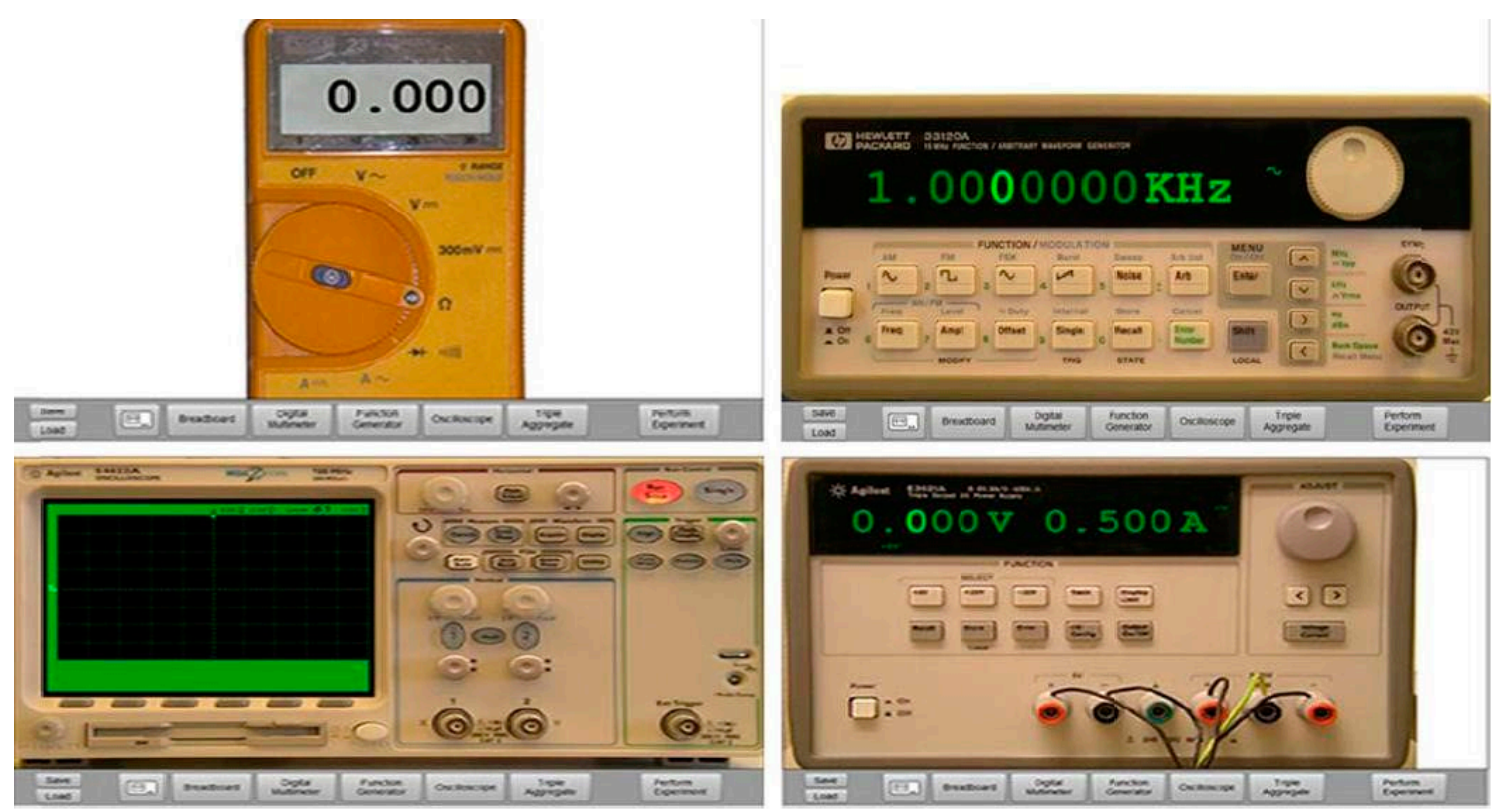

Fig. 3: Instrumentos del laboratorio remoto VISIR.

Se eligió el trabajo práctico denominado Módulo IV circuito RLC. El mismo posee una secuencia de elaboración conceptual y procedimental que guía al alumno en el proceso de aprendizaje en áreas de electrotecnia.

En la figura 4 muestra cómo se conectaron los elementos eléctricos en el protoboar virtual usando la guía de laboratorio mencionado anteriormente.

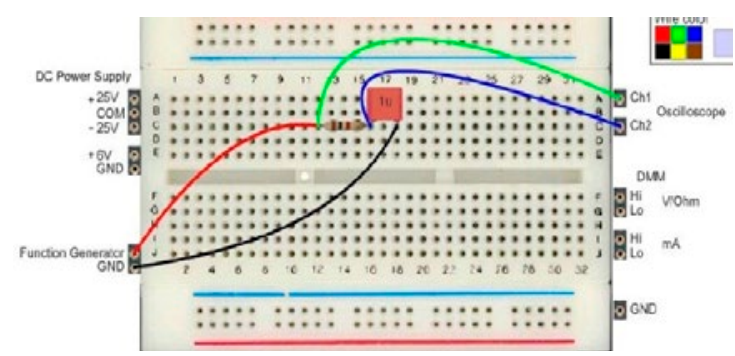

Fig. 4: Esquema virtual del circuito $R C$.

En esta última etapa el alumno contrasta las mediciones eléctricas reales con los valores teóricos. Se utilizan como fuente de información los datos obtenidos de los instrumentos del laboratorio remoto.

En la figura 5 se cuantifica mediante el osciloscopio la variación de la impedancia causada por efecto de la frecuenciaen el circuito RC de la figura 4.

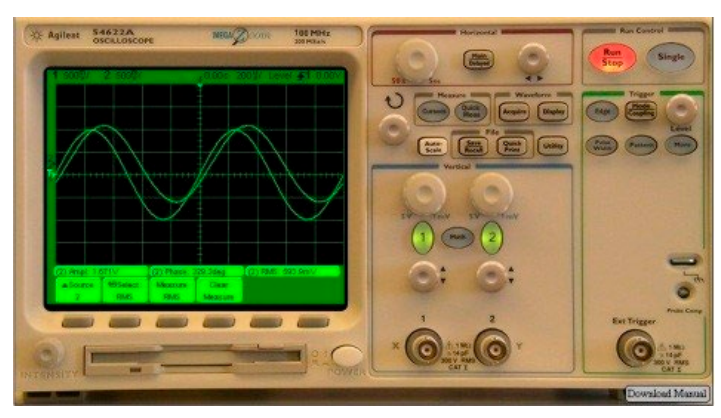

Fig. 5: Visualización y toma de datos empleando el osciloscopio.

Mediante la verificación y contrastación se logra internalizar conceptos eléctricos que fueron incorporados por un modelo físico en el área de electrotecnia. 
La guía de laboratorio tiene unas se- ejemplo, en la figura 6 y figura 7 se visualiza ries de tareas a realizar por parte del alum- una tabla y un gráfico donde se aprecian los no donde facilita la contrastación sobre los efectos de la frecuencia en el valor de la imresultados prácticos con los teóricos. Por pedancia en un circuito RC.

\begin{tabular}{|l|c|c|c|c|c|c|c|c|}
\hline Frecuencia Hz & 20 & 100 & 200 & 500 & 1000 & 2000 & 5000 & 10000 \\
\hline Impedancia Z1 & 79,577 & 15,915 & 7,958 & 3,183 & 1,592 & 0,796 & 0,318 & 0,159 \\
\hline Impedancia Z2 & 7,958 & 1,592 & 0,796 & 0,318 & 0,159 & 0,08 & 0,032 & 0,016 \\
\hline Impedancia Z3 & 0,796 & 0,159 & 0,08 & 0,032 & 0,016 & 0,007958 & 0,003183 & 0,001592 \\
\hline Impedancia R1 & 1 & 1 & 1 & 1 & 1 & 1 & 1 & 1 \\
\hline Impedancia R2 & 2 & 2 & 2 & 2 & 2 & 2 & 2 & 2 \\
\hline Impedancia Rg & 0,05 & 0,05 & 0,5 & 0,05 & 0,05 & 0,05 & 0,05 & 0,05 \\
\hline
\end{tabular}

Fig. 6: Valores de la impedancia en función de la frecuencia.

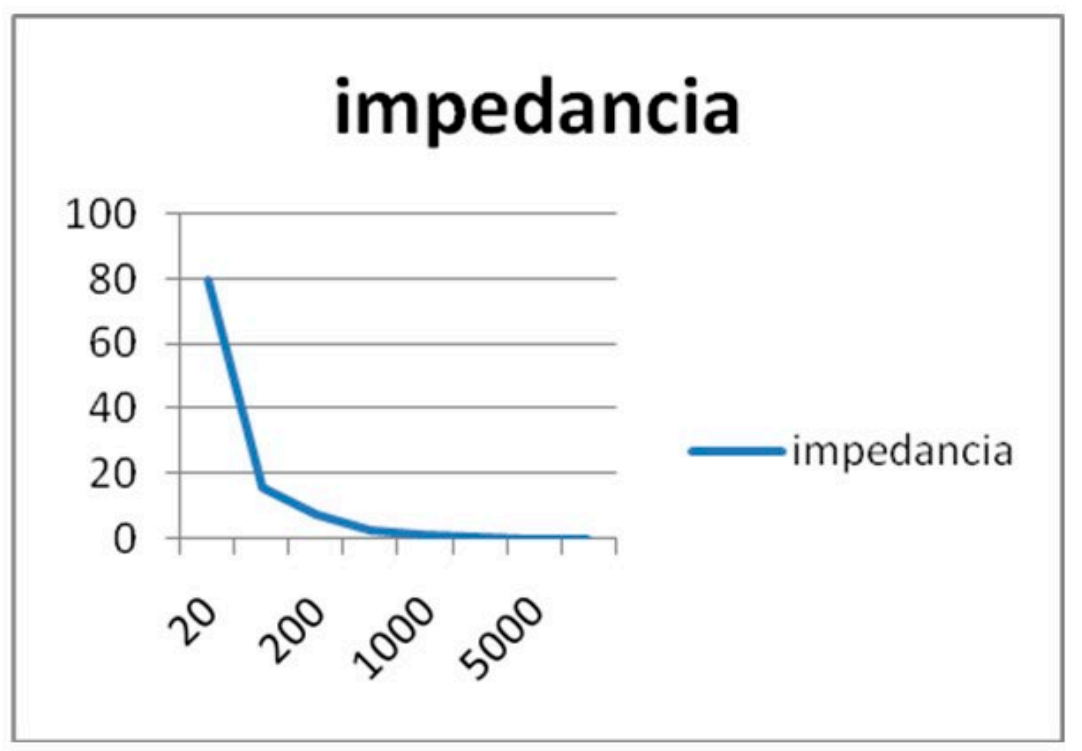

Fig. 7: Efectos de la frecuencia en la impedancia.

También es importante destacar que las variables eléctricas medidas por losinstrumentos en algunos casos difieren con la teoría debido a la naturaleza real de la experiencia.

\section{RESULTADO Y DISCUSIÓN}

Del análisis de resultados obtenidos de la guía de trabajos prácticos entregados por los alumnos permitieron inferir las siguientes conclusiones: a) Los aprendices mostraron interés, entusiasmo y compromiso con los nuevos conceptos incorporados durante el primer y segundo momento de la práctica.

b) La experiencia impactó positivamente en los aprendices no solo al favorecer la apropiación de conceptos abstractos sino también al facilitar la construcción de un sentido de aplicación de un objeto de estudio, que en este caso fue los efectos causados por la frecuencia en la impedancia RC. 
c) La mayoría de los estudiantes por primera vez visualizaron claramente la representación de un fenómeno físico usando un osciloscopio, en dominio del tiempo.

d) La mayoría de los estudiantes resaltaron el potencial didáctico que posibilita el poder indagar, experimentar, observar y contrastar el comportamiento de distintos tipos de señales y su respuesta variando la frecuencia del generador de frecuencia.

e) La experiencia contribuyó a una mayor articulación e integración entre los docentes de teoría y práctica.

f) Se favoreció la reflexión colectiva por parte de los docentes acerca del impacto pedagógico del proceso y se contribuyó a repensar y resignificar la práctica docente.

A continuación, se plantean los principales interrogantes que fueron surgiendo durante el proceso:

- ¿Se puede potenciar el autoaprendizaje del alumno utilizando este tipo de experiencias?

- ¿Cómo impacta en el rol docente?

- ¿Están los docentes preparados para incorporar este tipo de experiencias?

- ¿Qué competencias claves favorecen este tipo de experiencias?

- ¿Este tipo de prácticas puede complementarse con el aprendizaje basado en problemas?

- ¿Es fundamental la formación de un equipo sinérgico entre docentes, aprendices e ingenieros con experiencias en instrumentos y trabajo en laboratorios?

- ¿Cómo podría mejorar la articulación entre teoría y práctica utilizando esta nueva herramienta?

- ¿Estamos preparados para encarar este tipo de experiencias?

- ¿Qué necesitamos para llevarlas adelante?

- Este tipo de abordajes ¿se dan por la casualidad cuando los intereses de un grupo de docentes se encuentran y retroalimen$\tan$ ?

- ¿Es posible reproducirlas con otros temas y otros contextos? ¿Qué y Cómo sistematizar?

- ¿Existen buenas prácticas que se puedan seguir?

\section{IV.CONCLUSIONES}

Se describió una experiencia realizada en elárea de Electrotecnia de Ingeniería que se dicta en la Universidad Tecnológica $\mathrm{Na-}$ cional Facultad Regional Resistencia.

El objetivo principal fue facilitar el proceso de aprendizaje del alumno y mejorar la práctica docente mediante la utilización de estrategias basadas en la indagación y la experimentación. Para tales fines se articularon aspectos formales y teóricos de electricidad y fenómenos físicos observados en el mundo real.

Se partió del supuesto que al sujeto de aprendizaje que se inicia en la Ingeniería le resulta complejo relacionar leyes fundamentales como ley de Ohm o Kirchhoff. A fin de poder facilitar el aprendizaje de conceptos relacionado con electrotecnia elemental, se partió de un laboratorio previamente desarrollado por el VISIR que permitió cuantificar el comportamiento de circuitos eléctricos elementales en función 
de la frecuencia y contrastar los resultados obtenidos según lo predicho por el modelo físico teórico.

Esta modalidad de aprendizaje impactó tanto en el rol del docente como en el rol del alumno.

El docente se convirtió en un guía o asesor encargado de sugerir posibles caminos y soluciones a problemas planteados, por otro lado, debe diseñar material didáctico fuertemente orientado a la experimentación mediante el uso de instrumentos, dispositivos y herramientas de análisis y observación.

Se debió desarrollar habilidades relacionadas con el uso de ambientes de prueba y laboratorio, además de poder interactuar con profesionales en este campo.
Por otro lado, el alumno debió desarrollar competencias que le permitan indagar, analizar, experimentar, contrastar y aplicar resultados, de manera tal de poder apropiarse significativamente de nuevos conocimientos relacionados con el tema de estudio.

Esta modalidad le permitióal alumno el autoaprendizaje basado en la indagación y la comprobación de conceptos teóricos por medio de la experimentación.

\section{AGRADECIMIENTOS}

Se agradece a la Universidad Nacional de Santiago del Estero por su gentiliza en brindar información relevante que sirvió significativamente en la redacción de este trabajo.

\section{REFERENCIAS}

[1] Lerro F, Marchisio S, Von Pamel O. (2009). Exploring didactic possibilities of an electronic devices remote lab with students of Electronic Engineering. Conference ICBL2009. Florianopolis, Brazil.

[2] Lerro F, Marchino S, Plano M, Protano M, Von Pamel O. (2008). A remote lab like an educational resource in the teaching of the Physics of electronic devices. Conference ICBL2008. Villach, Austria.

[3] Marchisio S, Lerro F, Von Pamel O. (2010). Empleo y Aceptación por Estudiantes de Ingeniería de un Laboratorio Remoto de Dispositivos Electrónicos. SPDECE. Cadiz, España.

[4] Salah, R.; Alves, G.; Abdulazeez, D.; Guerreiro, P.; Gustavsson, I. (2015) Why VISIR? Proliferative Activities and Collaborative Work of VISIR System. 7th International Conference on Education and New Learning Technologies (EDULEARN15)
Proceedings, pp. 3824-3835.

[5] Concari, S.; Kofman, H.; Marchisio, S. (2012) Laboratorios remotos y educación en Ingeniería. Entre retos y posibilidades. XVII Congreso Internacional de Tecnologías para la Educación y el Conocimiento: Tecnologías Emergentes. UNED. España

[6] Marques, M.A.; Viegas, M.C.; Costa-Lobo, M.C.; Fidalgo, A.V.; Alves, G.R.; Rocha, J.S.; Gustavsson, I. (2014). How Remote Labs Impact on Course Outcomes: Various Practices Using VISIR. Education, IEEE Transactions, vol. 57, no. 3, pp. 151-159, Aug. 2014.

[7] Oguz-Unver A, Arabacioglu S (2014). A comparison of inquiry-based learning (IBL), problembased learning (PBL) and project-based learning (PJBL) in science education. Acad. J. Educ. Res. 2(7): $120-128$ 